\title{
In situ Observation of Annealing Effects in Ga(NAsP) Multi Quantum Well Structures
}

\author{
Rainer Straubinger ${ }^{1}$, Andreas Beyer $^{1}$, Lennart Duschek ${ }^{1}$, Tatjana Wegele ${ }^{1}$, Wolfgang Stolz ${ }^{1}$ and \\ Kerstin Volz ${ }^{1}$ \\ ${ }^{1 .}$ Philipps-Universität Marburg, Faculty of Physics and Materials Science Center, 35032 Marburg, \\ Germany
}

The epitaxial growth of multi component semiconductor layers such as $\mathrm{Ga}(\mathrm{NAsP})$ enables the improvement of laser and transistor devices due to the individually tunable band gap and lattice constant. Furthermore, the material system shows great potential for realizing optical light sources on Si substrate. Due to the low temperature growth conditions used for this metastable material system, point defects are incorporated in the material upon growth. In particular the thermal annealing procedure after growth can reduce the number of crystal defects and thus increase the efficiency of active regions of the devices [1]. One drawback of the thermal treatment is the gradual blur of the interfaces between the quantum wells and the barriers. To improve the understanding of the annealing process and in turn to improve the quality of the material system Ga(NAsP), (scanning) transmission electron microscopy ((S)TEM) investigations are indispensable. Especially the in situ observation of annealing effects during (S)TEM investigations can help to understand the physics behind these processes as many aspects of the formation of such complex materials are still unknown. Thereby the growth and annealing conditions can be optimized to improve the optical properties. From a more fundamental point of view, also the quantitative determination of the chemical composition of the quaternary alloy on an atomic scale as well as an understanding of the local effects like strain or atomic disorder on STEM contrast presents a true challenge, which is addressed in this study.

The investigated samples for these investigations were grown by metal organic vapor phase epitaxy (MOVPE) in a commercially available horizontal reactor system (AIX 200). Growth was performed with hydrogen as carrier gas at a temperature of $575^{\circ} \mathrm{C}$ and a reduced reactor pressure. $\mathrm{GaP} / \mathrm{Si}$ templates serves as pseudo substrate consisting of about $100 \mathrm{~nm}$ thick $\mathrm{GaP}$ layer nucleated on an exactly oriented $\mathrm{Si}(001)$ substrate. A more detailed description of the defect-free nucleation is given in Reference [2, 3]. For the in situ observation we use the atmosphere gas environmental cell from Protochips Inc. The environmental chip has nine electron transparent windows out of SiN with a diameter of around 10 micron. This allows us, in combination with our double Cs-corrected JEOL JEM $2200 \mathrm{FS}$ operating at $200 \mathrm{kV}$, to get high resolution images during the annealing process up to $1000^{\circ} \mathrm{C}$ under nitrogen environment. The images are acquired in scanning transmission electron microscopy (STEM) mode by using a high angle annular dark field detector (HAADF). Using the HAADF detector in combination with a highly focused beam which scans the sample column by column, the intensity of the final image is correlated to the atomic number of the element in the sample. This enables an intuitive way to distinguish between the different regions in the structure of the area of interest (compare figure $1 \mathrm{~B}, \mathrm{C}$ ). To realize this project the first step is to bring a electron transparent sample into the cell. Therefore we cut a regular 20 micron long lamella out of the sample using the focused ion beam (FIB) JIB $4601 \mathrm{~F}$ (JEOL) and prepare the area of interest with the H-bar method. This method has the advantage that the electron transparent part of the lamella is protected in between thicker regions. After the thinning process the lamella is lifted with the electron transparent part exactly over one of the $\mathrm{SiN}$ windows (compare figure $1 \mathrm{~A}$ ) and the lamella is fixed by using two tungsten depositions.

So far the ex situ observation using TEM has shown that there is a drastic change in the structure of 
the quantum well, after the annealing up to $975^{\circ} \mathrm{C}$. Figure 1 . B shows the cross section STEM image of the as grown $\mathrm{Ga}(\mathrm{NAsP})$ sample. The brighter line is the $\mathrm{Ga}(\mathrm{NAsP})$ quantum well embedded in $(\mathrm{BGa})(\mathrm{AsP})$. One can clearly see that there is no intensity fluctuation as well as no significant change in the width along the quantum well. Figure 1. C shows in comparison the same sample after the thermal treatment up to $975^{\circ} \mathrm{C}$. The destroyed area in the quantum well is marked with a black square. The quantum well shows a widening as well as a darker spot in the middle of the widening. At the moment it is not clear what the driving force for this change at the specific position in the quantum well is. It may be that the inhomogeneity of the composition or a thickness fluctuation triggers the change of the quantum well during thermal treatment.

As already mentioned the special combination of the atmosphere gas environmental cell and the in situ STEM observation allows us to investigate the thermal treatment at high resolution conditions in real time. These results as well as the quantification of the composition of the quaternary alloy will be presented and discussed [4].

References:

[1] S. Gies, et al, Journal of Crystal Growth, 402 (2014), p. 169.

[2] K. Volz, et al, Journal of Crystal Growth, 315 (2011), p. 37.

[3] B. Kunert, et al, Thin Solid Films, 517 (2008), p. 140.

[4] Support of the German Science Foundation (DFG) in the framework of the GRK 1782

("Functionalisation of semiconductors") is gratefully acknowledged.

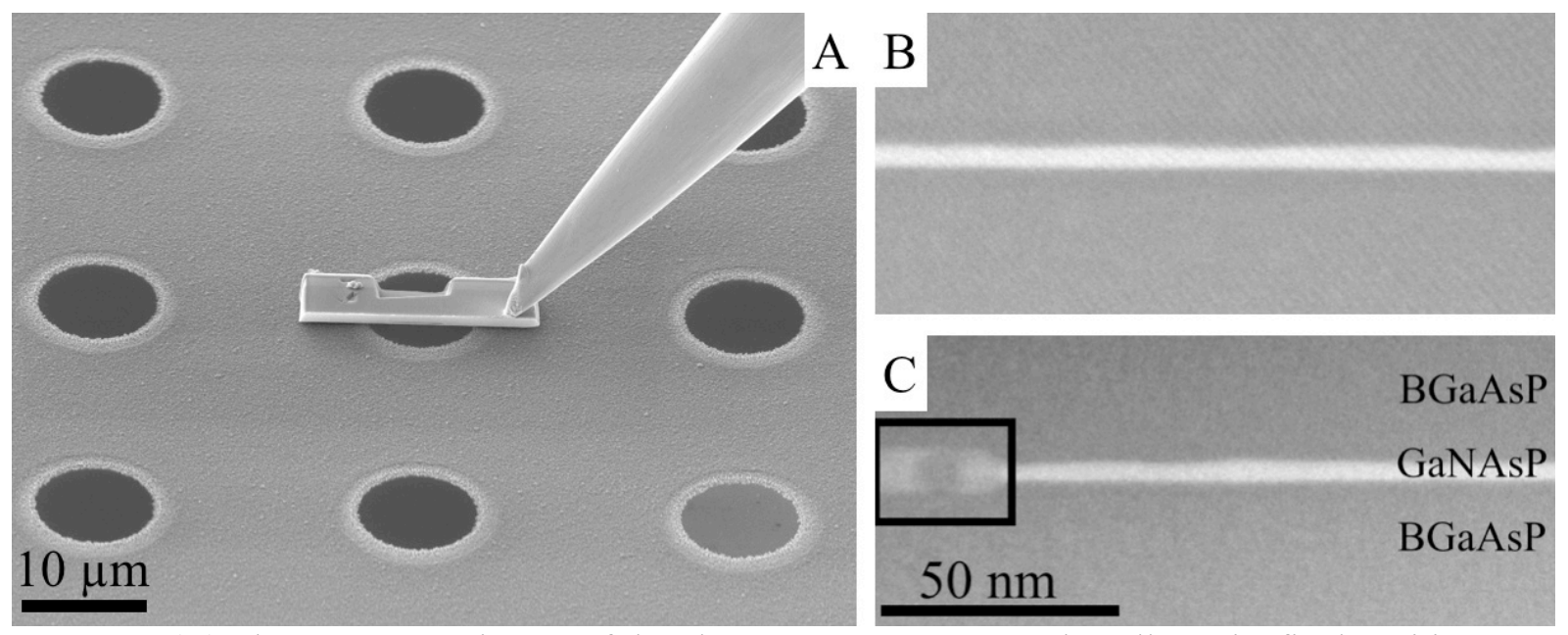

Figure 1. (A) Shows an SEM image of the electron transparent FIB lamella on its final position over the SiN window. (B) Cross section STEM image of the as grown and (C) annealed Ga(NAsP) quantum well structure. 